gen sollten. Doch mit welcher Substanz und wie lange sollte antikoaguliert werden? In der STENOX-Studie wurde das niedermolekulare Heparin (NMH) Enoxaparin in prophylaktischer und therapeutischer Dosierung über acht bis zehn Tage mit Placebo verglichen. Dabei fand sich ein „Catch-up-Effekt“, es kam also zu einer Aufholjagd, sodass der Nutzen des NMH nur in den ersten zwölf Tagen sichtbar, nach drei Monaten aber vollkommen aufgehoben war. In der CALIS-
TO-Studie wurde randomisiert 2,5 mg Fondaparinux einmal täglich über 45 Tage mit Placebo verglichen. Mit dieser Therapie ließ sich der primäre Endpunkt aus thromboembolischer Komplikation und Tod jeglicher Ursache um über $80 \%$ senken und zwar ohne Catch-up-Effekt. „Und bei dieser Therapie war das Blutungsrisiko nicht erhöht", so Dr. Florian Langer, Hamburg. Somit sei Fondaparinux bei der OVT die einzige evidenzbasierte Therapie.

\title{
Viele Medikamente machen Ödeme
}

\section{Bei der Abklärung von Beinödemen sollten Sie immer an medikamentöse Auslöser denken, ehe der Patient auf Herz und Niere untersucht wird.}

$B^{\mathrm{e}}$ ei Beinödemen wird zunächst an eine kardiale, renale oder venöse Ursache gedacht und eine entsprechende Abklärung veranlasst. „Doch oft wird vergessen, dass Medikamente die Übeltäter sind“, betonte Prof. Sebastian Schellong aus Dresden. Das Spektrum der dafür infrage kommenden Substanzen ist breit. Es reicht von Antihypertensiva über Hormone, NSAR bis hin zu Psychopharmaka.

\section{Häufig bei Kalziumantagonisten}

Bei den Kalziumantagonisten sind es vor allem Substanzen vom DihydropyridinTyp, wobei die der zweiten und dritten Generation wie Lercanidipin besser verträglich sind. Die Ödembildung betrifft vor allem Frauen und nimmt mit dem Alter zu. Pathophysiologisch entstehen Ödeme durch Dilatation der Arteriolen bei fehlender Wirkung auf Venolen. Es kommt zu einem präkapillären Leck. Aufgrund der Dosisabhängigkeit ist es sinnvoll, zunächst die Dosis zu senken, ehe man Patienten auf einen lipophilen Kalziumantagonisten der 3. Generation umstellt. Auch die Gabe am Abend oder die gleichzeitige Gabe eines RAAS-Blockers kann zur Rückbildung des Ödems beitragen. Sehr viel seltener können Ödeme bei Einnahme von Clonidin, Dihydralazin und Minoxidil auftreten.

\section{"Diuretika-Sucht"}

Bei den Diuretika sind es vor allem Schleifendiuretika wie Furosemid und
Torasemid, die Beinödeme auslösen können. Die Substanzen hemmen die Rückresorption von Natrium und Wasser, sodass beides vermehrt ausgeschieden wird. Kompensatorisch führt dies zu einem Anstieg von Aldosteron und des Antidiuretischen Hormons (ADH). Bei Nachlassen der Diuretikawirkung wird vermehrt Natrium und Wasser retiniert. „So entstehen Schwellung und Spannungsgefühl, was wiederum eine weitere, ja sogar verstärkte Einnahme des Diuretikums triggert“, erläuterte Schellong. Gerade bei jungen Frauen entwickelt sich nicht selten ein suchtartiges Einnahmeverhalten, das verleugnet wird. Es ist gar nicht so einfach, diese Patientinnen davon gänzlich zu entwöhnen.

\section{Angioödem bei ACE-Hemmern}

ACE-Hemmer machen zwar keine Beinödeme, können aber ein potenziell vital bedrohliches Angioödem auslösen. Die Inzidenz liegt bei ca. $1 \%$ und geht mit einer 1\%igen Mortalität einher. Die Komplikation tritt meist in den ersten vier Wochen nach Therapiebeginn auf, sie ist aber auch noch nach vielen Jahren möglich. Bei langwirksamen ACE-Hemmern verlaufen die Episoden schwerer, Diabetiker sind seltener betroffen. Auslöser ist das Bradykinin, dessen Abbau durch ACE-Hemmer ebenfalls gehemmt wird. Mildere Formen können als trockener Husten in Erscheinung treten. Obwohl es bei allen Patienten zu einem Bradykinin-Anstieg kommt, ist das Angioödem mit 1 auf 200 selten. „Warum nur wenige betroffen sind, wissen wir nicht“, so Schellong. Die Soforttherapie besteht in der Gabe von Adrenalin, Steroiden und Antihistaminika. „Der ACE-Hemmer muss abgesetzt werden und Betroffene sollten nie mehr einen solchen erhalten, auch keinen anderen“, betonte Schellong.

\section{Hormone, NSAR und}

\section{Psychopharmaka}

Alle Hormone können zu Wassereinlagerungen führen. Dies gilt besonders für weibliche Sexualhormone; sie haben Einfluss auf Gefäße, Wasserretention und Fettgewebe. Daher können sowohl physiologische als auch pathologische Hormonschwankungen die Wasserretention und Ödembildung verstärken. Besonders oft finden sich Ödeme bei Frauen mit polzystischem Ovar. Auch NSAR, unselektive wie selektive, können Ödeme induzieren. Ursache ist die Abschwächung der Prostaglandin-Wirkung auf die Niere bei Aufrechterhaltung der Natrium- und Wasserhomöostase. Das Ödem ist meist nur leicht und bildet sich nach Absetzen des Medikaments rasch zurück.

Bei den Psychopharmaka können Lithium, atypische Neuroleptika, MAOHemmer und trizyklische Antidepressiva Ödeme verursachen. Der Mechanismus ist unterschiedlich: Während Lithium mit Natrium bei der tubulären Rückresorption konkurriert, machen atypische Neuroleptika eine periphere Vasodilatation und senken so den peripheren Widerstand.

sti

Quelle: Jahrestagung der Deutschen, Österreichischen und Schweizerischen Gesellschaften für Angiologie und der Deutschen Gesellschaft für Phlebologie, 9.9.2016 in Dresden 